\title{
Biology of the Brain Disorders
}

National Cancer Institute

\section{Source}

National Cancer Institute. Biology of the Brain Disorders. NCI Thesaurus. Code C15880.

NIH Emphasis Area -- New emphasis on the biology of brain disorders reflects the extraordinary rate at which the neurosciences, with strong support from the fields of molecular genetics, imaging, and cell biology, is growing. Efforts in neural development; neurodegeneration, especially in Alzheimer's disease, Parkinson's disease, mental illness and traumatic injury to the brain and spinal cord are few examples of the promise of this field. 